\title{
Neuro-ophthalmological study of late yaws I. An introduction to yaws
}

\section{J. LAWTON SMITH}

From the Department of Ophthalmology, University of Miami School of Medicine, Miami, Florida, U.S.A.

Three major forms of treponemal disease are found in man: syphilis, due to Treponema pallidum; yaws, due to Treponema pertenue; and pinta, due to Treponema carateum. The spirochaetes that cause these diseases are morphologically indistinguishable. Furthermore, the diseases cannot be differentiated by any known serological test or therapeutic criterion. Only two ways are now known by which the treponematoses can be differentiated:

(1) Clinical criteria ;

(2) Experimental infection of laboratory animals or human volunteers.

The rising incidence of infectious syphilis in the United States, the usefulness of the FTA-ABS test in diagnosis of late 'seronegative' syphilis, and the detection of treponemes in human aqueous humour and cerebrospinal fluid have recently given impetus to the study of late ocular and neurosyphilis. Ocular and neurological involvement are common in late syphilis and are well recognized, but such involvement is said not to occur in either yaws or pinta. This contrast prompted a cooperative neuro-ophthalmological study of late yaws and pinta between the Department of Ophthalmology of the University of Miami, and the National Institute of Venereology in Caracas, Venezuela. Because of the lack of familiarity of most English-speaking physicians with the tropical, non-venereal treponematoses, the purpose of this report is to provide a brief introduction to yaws as a disease entity as obtained from the literature (Biggam and Wright, 1964; Felsenfeld, 1965) with emphasis on late aspects of the disease which are of neuroophthalmological interest.

Yaws, also known as pian or framboesia, is a disease of certain tropical countries in Central and South America, Africa, and the Far East. The causal agent, Treponema pertenue, discovered by Castellani (1905), is morphologically indistinguishable from the agents causing syphilis and pinta. Yaws occurs in patchy distribution in hot and humid regions. is Dark-skinned children under 10 years of age who $N$ live in rural areas are more commonly involved. 으 Poor hygiene and lack of personal cleanliness pre- $\underset{\neg}{\overrightarrow{2}}$ dispose to the disease. The incidence is slightly higher $\rightarrow$ in boys than girls, perhaps because of the higher incidence of skin abrasions in the former. The in- $\frac{5}{\omega}$ fection is spread by direct contact, and also by soiled clothing and bedding. According to the literature, yaws is not venereal and not hereditary. The disease is divided into primary, secondary, and tertiary stages, which are also designated initial, early, and late stages in the Hackett classification. Yaws is said to involve only the skin, mucous membranes, bones, and joints, and visceral lesions are said not to occur. Although monkeys, hamsters, and rabbits have been $\bar{F}$ infected with yaws experimentally, the only natural reservoir of the disease seems to be man.

\section{Clinical course}

The initial lesion, after an incubation period of 2 to 8 weeks, is called the 'mother yaw'. This is usually single and is found on parts of the body exposed to trauma, often the legs or buttocks. Non-immune mothers carrying children on their backs may develop lesions on the flanks from contact with yaws on the infants legs. The initial yaw begins as an erythe- $\frac{D}{0}$ matous papule that rapidly becomes granulomatous, and becomes covered with a crust of dried exudate. N The pinkish-yellowish lesion reaches 2 to $5 \mathrm{~cm}$. in N diameter in one or more weeks, and may remain solitary for 1 to 3 months. There is usually some degree of regional lymphadenopathy.

The secondary eruption usually begins 3 to 6 weeks later, sometimes before the primary lesion has healed. Papillomata, often occurring in crops, become very numerous in moist flexure surfaces and around the mouth. These lesions are highly contagious. Those around the mouth and nose are usually moist $\cong$

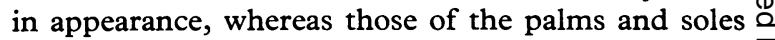
are dry and hard, often with numerous fissures and a tendency to ulcerate. If such a lesion erupts through $\delta$ the sole, walking becomes painful and the resultant? 
gait led to the term 'wet crab yaws'. Bony involvement often causes the fingers to become swollen and sausage-like. A similar involvement of the long bones may cause sabre tibia. Swelling of the nasal bones is called a goundou. Early mucosal yaws may be maculopapular or papillomatous. This is most commonly seen in continuity with skin lesions of mouth and nose. Other lesions of early yaws include a variety of skin eruptions, synovitis, periosteitis, hydrarthrosis, and diffuse ganglia.

Healing of the skin lesions begins with clearing of exudate, followed by loss of the crust. There is usually no scar, except after heavy secondary infection, but hyperpigmentation may result and may last for years.

\section{Laboratory studies}

The serological tests for syphilis, including both reagin (VDRL) and treponemal (TPI and FTAABS) tests, become reactive, and so do not help to differentiate syphilis, yaws, and pinta. Histopathological differentiation of the lesions may likewise be difficult, as the changes differ primarily in degree. The initial yaw and the secondary lesions, histologically, are proliferative granulomata with numerous treponemes. Syphilitic lesions are said to show only moderate acanthosis, no gross inflammation in the dermis, and proliferative changes in intima and media of vessels. In contrast, yaws lesions tend to show marked acanthosis, more marked inflammation in the dermis, but no proliferation of intima or media of vessels.

\section{Latent and late yaws}

After the spontaneous resolution of early yaws, there is usually a latent period during which serological changes may persist in the absence of clinical signs. Tertiary lesions usually develop 5 to 10 years after infection, but it should be noted that not all patients develop late yaws. The commonest manifestations of late yaws are cutaneous. Nodular lesions develop which ulcerate and spread superficially, and at times penetrate deeply into the underlying tissues. These can produce gross deformity. The lesions of late yaws tend to heal with scarring in one site, even while the ulcer is extending in another direction. Another late manifestation is hyperkeratosis with fissuring of the palms and soles, known as 'dry crab yaws'. The location of late lesions is of interest. Late yaws may develop on the site of the mother yaw. A mother with latent yaws nursing a child with early yaws of the face may develop a tertiary ulcer of the breast. These findings have suggested sensitivity and local re-infection as factors in late yaws.
Osseous changes in late yaws are important. Radiological examination of the long bones may show localized areas of rarefaction with surrounding periostitis. These may present clinically as localized bony swellings, and the overlying tissue may ulcerate and resemble a gumma. Bony involvement in the hands, outer table of skull, nose, and palate is not uncommon.

The milder cases, which are more common, may show a sunken nasal bridge or small palatal perforation. In extreme cases, gross mutilation may make the nose and mouth one open cavity (gangosa). Such lesions, with distortions of bones, contraction of joints, ulceration, and scarring, may produce a tragic clinical picture.

Other late manifestations of yaws include hydrarthrosis, bursitis, and firm, painless subcutaneous juxta-articular nodules, usually found near the elbows, hips, and knees.

From the neuro-ophthalmological point of view, yaws would appear from the consensus of the literature, to be an unrewarding disease to investigate. Biggam and Wright (1964) stated:

'Unlike tertiary syphilis, yaws does not affect the cardiovascular system or internal viscera, and the only neurological changes are very minor alterations of the cerebrospinal fluid'.

Medina (1963), the world authority on the disease, stated:

'Nowadays, it is agreed that only syphilis can bring about visceral changes, particularly of the nervous system and the aorta, and can establish itself in the placenta, thus giving rise to congenital syphilis. Yaws can never bring about these pathological conditions'.

The mention of minor alterations in the cerebrospinal fluid arouses interest. A few voices of dissent have been recorded. In a paper entitled 'Hereditary Yaws', Leon (1929) stated:

'In speaking today on the subject of congenital yaws, I am aware of the rashness of discussing a condition which is said not to exist both by the older classical medical texts and by more recent literature'.

Leon observed that Stitt (1922) had stated: 'It is quite obvious that there does not exist a congenital form of yaws' and that Roussel had remarked: 'Yaws is neither hereditary nor congenital'. However, Bertin (1786), after working in the French colony of San Domingo wrote that 'Yaws, as the venereal diseases and others, is transmitted through heredity'. Hunt and Johnston (1923), after studying 2,000 cases in Samoa, described interstitial keratitis in hereditary yaws. Leon (1929) concluded:

'That yaws may be hereditary is possible. Fetal mortality appears to be about five times greater in syphilitic than yaws cases'. 
The classic studies of Medina (1963), utilizing experimental inoculations of treponemes into human volunteers and studying the immune status of patients with syphilis, yaws, pinta, and controls, are so important that anyone interested in the nonvenereal treponematoses should read these in substance. Electron microscopical studies of the treponemes are pertinent. Ovcinnikov and Delektorskij (1969), comparing the ultrastructure of Treponema pertenue and Treponema pallidum, reported that, although structural differences did exist, they failed to find any reliable structural criteria by which to distinguish these species.

Finally, long-acting penicillin therapy has been reported as highly effective in public health anti-yaws campaigns. These have been primarily concerned with the active, early cases. Public health measures, as well as emphasis on sanitation and hygiene, have been effective in reducing the magnitude of the yaws problem, but have not eradicated the disease.

The reported persistence of Treponema pallidum in patients with late seronegative syphilis despite large doses of penicillin therapy makes the investigation of patients with late yaws and pinta of great interest. Such a study is presented in the following report.

\section{References}

BERTIN, N. (1786) 'Des moyens de conserver la santé des blancs et des nègres aux Antilles du climats chauds et humides de l'Amérique ...' Méquignon, Paris.

BigGaM, A., and WRIGHT, F. J. (1964) 'Tropical Diseases', ed. S. Davidson, p. 1299. Livingstone, Edinburgh (Supplement to 'The Principles and Practice of Medicine', 7th ed., by S. Davidson)

Botreay-Roussell: 'Médecin des troupes coloniales. Infections à germe connues', in 'Traité de pathologie médicale et de thérapeutique appliquée', par E. Sergent, etc., 33 vols (cited by Leon, 1929)

Castellani, A. (1905) Brit. med. F., 2, 1280, 1330, 1430

Felsenfeld, O. (1965) 'Synopsis of Clinical Tropical is Medicine: Pathogenesis, Clinical Picture, Diag- N nosis, Prognosis, and Therapy', p. 93. Mosby, St. Louis

Hunt, D., and Johnson, A. L. (1923) U.S. nav. med. Bull., 18, 599

Leon, R. (1929) Amer. F. trop. Med., 9, 439

MEDiNA, R. (1963) WHO/VDT/RES 63.64

OvcinNikov, N. M., and DelektorskiJ, V. V. (1969) WHO/VDT/RES 69.184

StitT, E. R. (1922) 'The Diagnosis and Treatment of Tropical Diseases', 4th ed. Lewis, London (cited by Leon, 1929) 